\title{
A INFLUÊNCIA DOS PARÂMETROS DE CALIBRAÇÃO DE ANTENAS GPS NAS ALTITUDES ELIPSOIDAIS NO POSICIONAMENTO RELATIVO ESTÁTICO
}

\section{The influence of GPS antennas calibration parameters in ellipsoidal heights in relative static positioning}

\author{
Renata Magda Cavalcante Werlich ${ }^{1}$ \\ Claudia Pereira Krueger ${ }^{1}$ \\ Günter Schmitt ${ }^{2}$
}

Universidade Federal do Paraná ${ }^{1}$ Departamento de Geomática.Caixa Postal 19001 - Centro Politécnico Jardim das Américas - 81531-990 Curitiba/PR, Brasil. renatawerlich@gmail.com; ckrueger@ufpr.br Karlsruher Institut für Technologie ${ }^{2}$ Geodästisches Institut. Englestraße 7 - 76131 Karlsruhe, Deutschland guenter.schmitt@kit.edu

\section{Resumo:}

Alta acurácia em posicionamento GPS (Global Positioning System) é obtida através da eliminação parcial ou total dos erros presentes nas observações, particularmente os sistemáticos. Um deles é o erro de centro de fase da antena GPS. Ele é diretamente dependente da antena utilizada, visto que cada uma delas tem suas características próprias de construção e recepção do sinal GPS. Portanto, é de extrema importância o conhecimento individual do centro de fase da antena (Phase Center Offset - Phase Center Variation). Entre outros, existem parâmetros fornecidos pelo NGS (National Geodetic Survey), no entanto, esses valores são médios e não são valores próprios das antenas usadas nos posicionamentos GPS. Os parâmetros individuais das antenas, neste trabalho, foram fornecidos pela BCAL/UFPR (Base de Calibração de Antenas GNSS na UFPR). Realizaram-se posicionamentos GPS em 9 (nove) pontos. Estes foram distribuidos em 3 (três) grupos de linhas de base $(9 \mathrm{~km}, 45 \mathrm{~km}$ e $105 \mathrm{~km})$. Para cada comprimento de linha de base os rastreios GPS seguiram simultâneos aplicando o Método de Posicionamento Relativo Estático, com um intervalo de gravação de dados de 15 segundos e máscara de elevação de $15^{\circ}$. Foram encontradas diferenças na comparação das altitudes elipsoidais e no comprimento das linhas de bases, quando não se utilizou parâmetros, na ordem do centímetro. De fato, os valores obtidos da BCAL/UFPR são provenientes da utilização dos próprios equipamentos, desta forma, fiéis às antenas utilizadas, enquanto que os fornecidos pelo NGS são valores médios. Destaca-se que a BCAL/UFPR é a primeira base de calibração de antenas GPS/GLONASS no Brasil e na América Latina. 
Palavras-chave: Sistema de Posicionamento Global, Alta acurácia, Calibração de Antena, Posicionamento, BCAL/UFPR.

\begin{abstract}
:
High accuracy in GPS (Global Positioning System) positioning is obtained by partial or total errors eliminating present in the observations, particularly the systematic ones. One of them is called GPS antenna phase center error. It is directly dependent on the antenna used because each antenna has its own design characteristics and GPS signal reception. Therefore, it is extremely important to know the individual antenna phase center variation (Phase Center Offset - Phase Center Variation). There are parameters provided by the NGS (National Geodetic Survey), however, they are mean values and not individual values of the antennas used in GPS positioning. In this work individual parameters were provided by BCAL/UFPR (Base de Calibração de Antenas GNSS na UFPR). GPS surveys were carried out in nine (9) points. These were divided into three (3) baselines groups $(9 \mathrm{~km}, 45 \mathrm{~km}$ and $105 \mathrm{~km})$. For each baseline length the GPS surveys were simultaneous and the Method Static Relative Positioning was applied, with an interval of data recording of 15 seconds and $15^{\circ}$ elevation mask. Differences up to one centimeter were found when comparing the ellipsoidal heights and baselines length obtained using the BCAL/UFPR parameters and the NGS ones. In fact, the values obtained with the BCAL/UFPR parameters are derived using own parameters to the antennas used, whereas those provided by the NGS are mean values. It is noteworthy that the BCAL/UFPR is the first antenna calibration site GPS/GLONASS in Brazil and Latin America.
\end{abstract}

Keywords: Global Positioning System, High Accuracy, Antenna Calibration, Positioning, BCAL/UFPR.

\title{
1. Introdução
}

O posicionamento GPS possibilita o conhecimento da localização de qualquer objeto sobre a superfície da Terra. Desta forma, cabe ressaltar, que dependendo do equipamento, técnica empregada e da estratégia de processamento, torna-se possível determinar coordenadas geodésicas tridimensionais com acurácia relativa que varia de metros até milímetros.

Aplicações geodésicas que demandam alta acurácia requerem uma atenção especial aos erros sistemáticos. Tais aplicações podem se basear em redes de monitoramento contínuo, como por exemplo, a RBMC (Rede Brasileira de Monitoramento Contínuo dos Sistemas GNSS), em monitoramento de estruturas civis, dentre outras.

Ao lado dos erros troposféricos e do efeito de multicaminho, a variação do centro de fase da antena é um fator limitante na determinação de coordenadas geodésicas precisas (Wübbena et al., 1996). Cada antena tem suas características individuais, consequentemente, as variações são singulares e desta forma, devem ser tratadas de forma particular (Kersten e Schön, 2010). Os valores para a correção do erro de centro de fase da antena são os PCO (Phase Center Offset) e PCV (Phase Center Variation).

No Brasil, diferentemente da Europa, os usuários não têm o costume de aplicar os valores individuais de calibração da antena para minimizar o erro causado pelo seu centro de fase. 
Quando estes usuários empregam os valores de calibração das antenas, eles utilizam, em geral, os valores médios fornecidos pelo NGS (National Geodetic Survey) (Santos et al., 2010, Pinto et al., 2015 e Silva et al. 2013), fato este que pode conduzir a erros sistemáticos no posicionamento de alta acurácia. Ignorar os valores das variações do centro de fase da antena, na determinação de coordenadas geodésicas precisas de um ponto, pode conduzir a erros na coordenada vertical de até $10 \mathrm{~cm}$ (Mader, 1999). Nas coordenadas horizontais pode-se introduzir erros de até $3 \mathrm{~cm}$ (Seeber, 2000).

No presente trabalho, almeja-se aplicar em levantamentos com o GPS antenas calibradas individualmente na BCAL/UFPR. As calibrações realizadas nessa base seguem a metodologia da calibração relativa. No entanto, são gerados parâmetros em nível absoluto. Serão utilizados três diferentes comprimentos de linhas de base, com o intuito de investigar a importância do emprego dos parâmetros próprios de calibração de antenas para os posicionamentos de alta acurácia. Para possibilitar uma melhor investigação quanto à contribuição da utilização dos parâmetros, em cada comprimento de linha de base os rastreios foram simultâneos. Isto implicou em uma geometria de satélites similar em cada um dos pontos rastreados.

\section{Metodologia}

\section{1 Área de Estudo}

A presente pesquisa engloba a BCAL/UFPR, localizada no Campus Jardim das Américas do Centro Politécnico da Universidade Federal do Paraná, Curitiba, Paraná, e uma área ao seu entorno com raio de até $105 \mathrm{~km}$.

Esta área de estudo (Figura 1) foi subdividida em 3 grupos:

- $9 \mathrm{~km}$ de raio a partir da BCAL/UFPR - primeiro círculo na Figura 1, próximo a BCAL/UFPR;

- $45 \mathrm{~km}$ de raio a partir da BCAL/UFPR - segundo círculo na Figura 1; e

- $105 \mathrm{~km}$ de raio a partir da BCAL/UFPR - ainda na Figura 1, terceiro círculo.

Respectivamente, os pontos pertencentes ao raio de $9 \mathrm{~km}$ são denominados 1,2 e 3; ao raio de 45 km são denominados 4, 5 e 6; e ao raio de 105 km são denominados 7, 8 e 9 . 


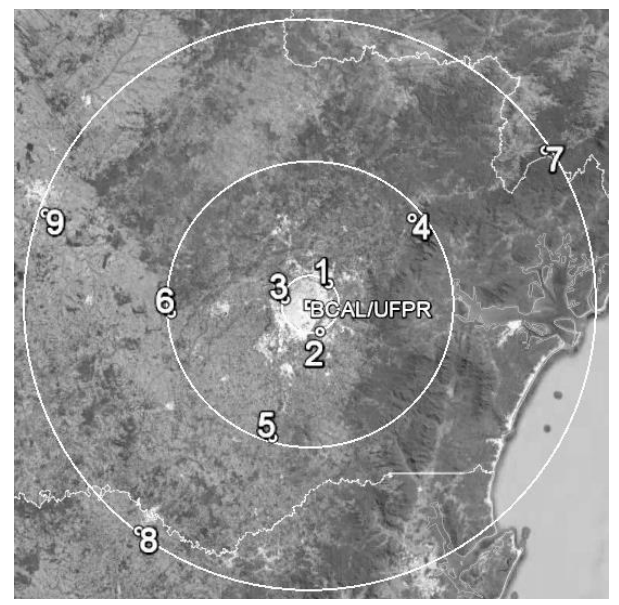

Figura 1: Área de estudo. Fonte: Adaptado de Google Earth 2014.

\subsection{Equipamentos}

Visando à determinação das coordenadas geodésicas da estação utilizada como estação base (Pilar 2000 pertencente à BCAL/UFPR) (Figura 1) e dos pontos com linhas de base curtas, médias e longas, foram utilizadas as antenas GPS apresentadas na Tabela 1. Os receptores GPS empregados são da série 1200, da Leica.

Tabela 1: Antenas GPS utilizadas.

\begin{tabular}{c|c}
\hline Pontos & Antena (Modelo / número de série) \\
\hline 1,4 e 8 & LEIATX 1230/155949 \\
\hline 2,5 e 9 & LEIAX 1202 GG / 8490007 \\
\hline 3,6 e 7 & LEIAX 1203+ GNSS / 9030001 \\
\hline Pilar 2000 & LEIAR25 / 09050004 \\
\hline
\end{tabular}

A antena instalada no Pilar 2000 é uma antena ChokeRing 3D que possibilita a minimização do efeito de multicaminho. Isto ocorre devido às depressões e distâncias entre os anéis concêntricos na estrutura da antena. Esta antena foi calibrada utilizando o método absoluto em campo pela empresa Geo++ (Gesellshaft für satellitengestützte und navigatorische Technologien mbH). Neste método de calibração a antena a ser calibrada é acoplada a um robô que realiza diversas rotações na antena. Ele tem como vantagem não empregar uma antena de referência (Menge et $a l$, 1998). Os valores de PCO, para as ondas portadoras $\mathrm{L}_{1} \mathrm{e} \mathrm{L}_{2}$, da antena LEIAR25 são apresentados no Tabela 2.

Tabela 2: Offsets da antena LEIAR25

\begin{tabular}{c|c|c|c|c}
\hline \multirow{2}{*}{} & \multicolumn{5}{|c}{ PCO ABSOLUTO DA GEO++ } \\
\cline { 2 - 5 } & PCO L1 - Norte $(\mathrm{mm})$ & 1,26 & PCO L2 - Norte $(\mathrm{mm})$ & 0,26 \\
\cline { 2 - 5 } & PCO L1 - Leste $(\mathrm{mm})$ & $-1,38$ & PCO L2 - Leste $(\mathrm{mm})$ & $-1,23$ \\
\cline { 2 - 5 } & PCO L1 - Vertical $(\mathrm{mm})$ & 159,50 & PCO L2 - Vertical $(\mathrm{mm})$ & 162,26 \\
\hline
\end{tabular}




\subsection{Coleta e Processamentos dos Dados GPS}

As observações GPS foram coletadas com o Método de Posicionamento Relativo Estático, com os equipamentos descritos na seção 2.2. O intervalo de gravação dos dados foi igual a 15 segundos, com duração dos rastreios de 6 horas.

Com o objetivo de se obter condições semelhantes nos rastreios, foram determinados alguns prérequisitos para a escolha do local para a implantação dos novos pontos. São eles:

- Discrepância máxima nas linhas de base de $15 \%$ do comprimento, para que as linhas de base apresentem maior similaridade possível:

o $1,35 \mathrm{~km}$ para linha de base de até $9 \mathrm{~km}$;

o $6,75 \mathrm{~km}$ para linha de base de até $45 \mathrm{~km}$; e

o $15,75 \mathrm{~km}$ para linha de base de até $105 \mathrm{~km}$.

- Altitude elipsoidal entre $1.225 \mathrm{~m}$ e $625 \mathrm{~m}$, para que os efeitos atuantes da troposfera sejam similares;

- Livre de obstruções; e

- Fácil acesso.

Para a determinação das coordenadas geodésicas dos pontos 1 a 9, utilizando os diversos parâmetros de calibração a serem testados, foi empregado o programa Leica Geo Office. A estação de referência foi o Pilar 2000 (Norte), pertencente à BCAL/UFPR.

As configurações utilizadas nos processamentos foram:

- Efemérides pós-processadas finais do IGS (The International GNSS Service), (Kouba, 2009);

- Máscara de elevação de $15^{\circ}$;

- Para a linha de base de até $9 \mathrm{~km}$ :

o Sem a inserção de modelos troposféricos e ionosféricos;

o Combinação linear $\mathrm{L}_{1}+\mathrm{L}_{2}$.

- Para as linhas de base de até 45 e $105 \mathrm{~km}$ :

o Modelo Troposférico de Hopfield, (Seeber, 2003); e

o Combinação linear Ion-free $\left(\mathrm{L}_{3}\right)$ e $\mathrm{L}_{1}+\mathrm{L}_{2}$.

A combinação linear $\mathrm{L}_{1}+\mathrm{L}_{2}$ foi empregada para uma rápida solução das ambiguidades nas linhas de bases curtas e médias, enquanto que a combinação $\mathrm{L}_{3}$, adequada para linhas de bases maiores, foi empregada nas linhas de bases médias e longas. Foram criados quatro processamentos distintos, sem e com a inserção dos parâmetros de calibração (PCO-PCV). O primeiro, sem aplicar qualquer parâmetro de calibração. No segundo foram inseridos, para as três antenas utilizadas, os parâmetros médios absolutos do NGS. Ressalta-se que somente para a antena LEIATX 1230 os parâmetros absolutos disponibilizados no site do NGS são, na verdade relativos convertidos para absolutos. O terceiro processamento incluiu os valores médios relativos de PCO indicados pelo NGS. No quarto processamento foram inseridos os parâmetros gerados na BCAL/UFPR. É adequado lembrar que estes parâmetros foram determinados por uma calibração pelo método relativo em campo, porém, determinados em nível absoluto, visto que a antena de referência foi calibrada utilizando o método absoluto em campo (Seção 2.2). Os Tabelas 3, 4 e 5 sintetizam os valores de PCO empregados nos processamentos, a menos dos sem 
a inserção de parâmetros de calibração, para as antenas, respectivamente, LEIAX 1202 GG, LEIATX 1230 e LEIAX 1203+ GNSS. Aqui não serão apresentados os valores de PCV.

Tabela 3: Offsets da antena LEIAX 1202 GG

\begin{tabular}{|c|c|c|c|c|}
\hline \multirow{12}{*}{ 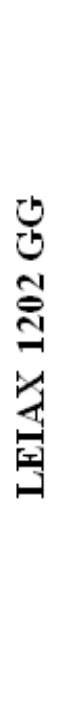 } & \multicolumn{4}{|c|}{ PCO ABSOLUTO DO NGS } \\
\hline & PCO L1 - Norte $(\mathrm{mm})$ & 1,64 & PCO L2 - Norte (mm) & $-1,20$ \\
\hline & PCO L1 - Leste (mm) & $-0,52$ & PCO L2 - Leste (mm) & 0,23 \\
\hline & PCO L1 - Vertical (mm) & 63,42 & PCO L2 - Vertical (mm) & 62,13 \\
\hline & \multicolumn{4}{|c|}{ PCO RELATIVO DO NGS } \\
\hline & PCO L1 - Norte (mm) & 1,60 & PCO L2 - Norte (mm) & $-1,20$ \\
\hline & PCO L1 - Leste (mm) & $-0,50$ & PCO L2 - Leste (mm) & 0,20 \\
\hline & PCO L1 - Vertical (mm) & 63,40 & PCO L2 - Vertical (mm) & 62,10 \\
\hline & \multicolumn{4}{|c|}{ PCO RELATIVO EM NÍVEL ABSOLUTO DA BCAL/UFPR } \\
\hline & PCO L1 - Norte (mm) & 1,36 & PCO L2 - Norte (mm) & $-0,51$ \\
\hline & PCO L1 - Leste (mm) & $-0,58$ & PCO L2 - Leste (mm) & 0,31 \\
\hline & PCO L1 - Vertical (mm) & 63,39 & PCO L2 - Vertical (mm) & 64,17 \\
\hline
\end{tabular}

Tabela 4: Offsets da antena LEIATX 1230

\begin{tabular}{c|c|c|c|c}
\hline & \multicolumn{5}{c}{ PCO ABSOLUTO (RELATIVO CONVERTIDO EM } \\
ABSOLUTO) DO NGS \\
\cline { 2 - 5 } & PCO L1 - Norte (mm) & 0,98 & PCO L2 - Norte (mm) & 0,02 \\
\cline { 2 - 5 } & PCO L1 - Leste (mm) & 2,13 & PCO L2 - Leste (mm) & $-1,09$ \\
\cline { 2 - 5 } & PCO L1 - Vertical (mm) & 92,45 & PCO L2 - Vertical (mm) & 97,25 \\
\cline { 2 - 5 } & \multicolumn{4}{|c}{ PCO RELATIVO DO NGS } \\
\cline { 2 - 5 } & PCO L1 - Norte (mm) & 1,00 & PCO L2 - Norte (mm) & 0,00 \\
\cline { 2 - 5 } & PCO L1 - Leste (mm) & 2,10 & PCO L2 - Leste (mm) & $-1,10$ \\
\cline { 2 - 5 } & PCO L1 - Vertical (mm) & 92,40 & PCO L2 - Vertical (mm) & 97,30 \\
\cline { 2 - 5 } & PCO RELATIVO EM NÍVEL ABSOLUTO DA BCAL/UFPR \\
\hline & PCO L1 - Norte (mm) & 1,33 & PCO L2 - Norte (mm) & $-0,42$ \\
\cline { 2 - 5 } & PCO L1 - Leste (mm) & 0,77 & PCO L2 - Leste (mm) & 2,26 \\
\cline { 2 - 5 } & PCO L1 - Vertical (mm) & 87,78 & PCO L2 - Vertical (mm) & 89,73 \\
\hline
\end{tabular}


Tabela 5: Offsets da antena LEIAX 1203+ GNSS

\begin{tabular}{|c|c|c|c|c|}
\hline \multirow{12}{*}{ 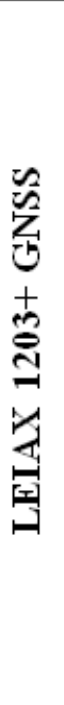 } & \multicolumn{4}{|c|}{ PCO ABSOLUTO DO NGS } \\
\hline & PCO L1 - Norte (mm) & $-1,03$ & PCO L2 - Norte (mm) & $-0,20$ \\
\hline & PCO L1 - Leste (mm) & 1,35 & PCO L2 - Leste (mm) & $-2,38$ \\
\hline & PCO L1 - Vertical (mm) & 58,32 & PCO L2 - Vertical (mm) & 55,54 \\
\hline & \multicolumn{4}{|c|}{ PCO RELATIVO DO NGS } \\
\hline & PCO L1 - Norte (mm) & $-1,00$ & PCO L2 - Norte (mm) & $-0,20$ \\
\hline & PCO L1 - Leste (mm) & 1,40 & PCO L2 - Leste (mm) & $-2,40$ \\
\hline & PCO L1 - Vertical (mm) & 58,30 & PCO L2 - Vertical (mm) & 55,50 \\
\hline & \multicolumn{4}{|c|}{ PCO RELATIVO EM NÍVEL ABSOLUTO DA BCAL/UFPR } \\
\hline & PCO L1 - Norte (mm) & $-0,96$ & PCO L2 - Norte (mm) & $-0,08$ \\
\hline & PCO L1 - Leste (mm) & 1,24 & PCO L2 - Leste (mm) & $-2,57$ \\
\hline & PCO L1 - Vertical (mm) & 59,38 & PCO L2 - Vertical (mm) & 55,97 \\
\hline
\end{tabular}

\section{Resultados}

Após o processamento dos dados GPS, verificou-se que os pré-requisitos (Seção 2.3) foram atendidos. Para o ponto 8 observou-se a necessidade de efetuar um novo processamento com a exclusão de 4 satélites. Isto ocorreu devido ao alto valor de resíduos para alguns satélites. Desta forma, obtiveram-se as altitudes elipsoidais sem e com a inserção de diferentes parâmetros de calibração das antenas. A Tabela 6 apresenta um resumo com as principais informações dos processamentos realizados. As informações listadas são comuns para os 4 processamentos realizados.

Percebe-se que para todos os pontos observados conseguiu-se uma solução fixa ou flutuante para as ambiguidades. Para as linhas de bases curtas e médias (em torno de $9 \mathrm{~km}$ e $45 \mathrm{~km}$ ), a combinação linear $\mathrm{L}_{1}+\mathrm{L}_{2}$ possibilitou estimativa fixa. Para as linhas de bases médias (nova solução) e grandes (em torno de $105 \mathrm{~km}$ ), a combinação linear L3 possibilitou estimativa flutuante. 
Tabela 6: Informações dos processamentos.

\begin{tabular}{|c|c|c|c|c|}
\hline Ponto & $\begin{array}{l}\text { Número de } \\
\text { satélites GPS }\end{array}$ & $\begin{array}{l}\text { Número de satélites } \\
\text { GPS exclúdos }\end{array}$ & $\begin{array}{l}\text { Solução da } \\
\text { ambiguidade }\end{array}$ & $\begin{array}{c}\text { Combinação } \\
\text { linear }\end{array}$ \\
\hline 1 & 15 & ----- & \multirow{3}{*}{ Fixa } & \multirow{3}{*}{$\mathrm{L}_{1}+\mathrm{L}_{2}$} \\
\hline 2 & 15 & ----- & & \\
\hline 3 & 14 & ---- & & \\
\hline 4 & 15 & ----- & \multirow{3}{*}{ Flutuante } & \multirow{3}{*}{$\mathrm{L}_{3}$} \\
\hline 5 & 15 & ----- & & \\
\hline 6 & 15 & ----- & & \\
\hline 4 & 15 & ----- & \multirow{3}{*}{ Fixa } & \multirow{3}{*}{$\mathrm{L}_{1}+\mathrm{L}_{2}$} \\
\hline 5 & 15 & ----- & & \\
\hline 6 & 15 & ---- & & \\
\hline 7 & 16 & ----- & \multirow{3}{*}{ Flutuante } & \multirow{3}{*}{$\mathrm{L}_{3}$} \\
\hline 8 & 16 & 4 & & \\
\hline 9 & 16 & ----- & & \\
\hline
\end{tabular}

Neste trabalho o efeito de multicaminho foi quantificado com o emprego do programa TEQC (Translate Edit Quality Check) da University Navstar Consortium (UNAVCO, 2010). Estes resultados indicam a qualidade dos dados com relação a este efeito. No entanto, ele não tem correlação com as análises realizadas, já que seu efeito é o mesmo para qualquer parâmetro de calibração de antena GPS utilizado.

$\mathrm{Na}$ Tabela 7 constam os valores de efeito de multicaminho para as portadoras $\mathrm{L}_{1}\left(\mathrm{MP}_{1}\right)$ e $\mathrm{L}_{2}$ $\left(\mathrm{MP}_{2}\right)$, quando inserida a máscara de elevação empregada no processamento dos dados GPS $\left(15^{\circ}\right)$.

Tabela 7: Efeito de multicaminho.

\begin{tabular}{|c|c|c|c|}
\hline \multirow{2}{*}{ Ponto } & \multirow{2}{*}{ Antena } & \multicolumn{2}{|c|}{ Máscara de elevação de $15^{\circ}$} \\
\hline & & $\mathrm{MP}_{1}$ (m) & $\mathrm{MP}_{2}$ (m) \\
\hline 1 & \multirow{3}{*}{ LEIATX 1230} & 0,238 & 0,273 \\
\hline 4 & & 0,179 & 0,186 \\
\hline 8 & & 0,245 & 0,325 \\
\hline 2 & \multirow{3}{*}{ LEIAX 1202 GG } & 0,260 & 0,299 \\
\hline 5 & & 0,150 & 0,165 \\
\hline 9 & & 0,266 & 0,330 \\
\hline 3 & \multirow{3}{*}{$\begin{array}{l}\text { LEIAX } 1203+ \\
\quad \text { GNSS }\end{array}$} & 0,219 & 0,307 \\
\hline 6 & & 0,756 & 1,519 \\
\hline 7 & & 0,279 & 0,326 \\
\hline
\end{tabular}

No ponto 6 foi verificado que o efeito de multicaminho divergiu dos demais pontos. Isto, provavelmente, ocorreu devido a um caminhão que ficou estacionado próximo à antena GPS. Os outros pontos apresentaram valores semelhantes em ambas as portadoras. 


\subsection{Análise das Altitudes Elipsoidais}

Os parâmetros obtidos na BCAL/UFPR foram tomados como referência visando à determinação das coordenadas geodésicas precisas dos pontos, visto retratam a situação de cada antena individual, e não valores médios para o tipo correspondente de antena (Huinca, 2009; Huinca e Krueger, 2011 e Huinca et al., 2012).

Foram determinadas as coordenadas geodésicas dos pontos, referenciadas ao SIRGAS2000, nos quatro processamentos: sem parâmetros de calibração; com parâmetros do NGS relativos; com parâmetros do NGS absolutos; e com parâmetros da BCAL/UFPR. Calcularam-se ainda as diferenças das altitudes elipsoidais entre as seguintes soluções: sem parâmetros e a BCAL/UFPR; NGS relativo e a BCAL/UFPR; e NGS absoluto e a BCAL/UFPR.

A Tabela 8 apresenta os resultados obtidos para as linhas de bases curtas (em torno de $9 \mathrm{~km}$ ). Verificam-se as altitudes elipsoidais dos pontos 1, 2 e 3 e as diferenças calculadas entre os resultados obtidos.

Tabela 8: Altitudes elipsoidais e as diferenças calculadas para os pontos situados em torno de 9 $\mathrm{km}$ de distância.

\begin{tabular}{c|c|c|c}
\hline Ponto & Parâmetro & Altitude elipsoidal (m) & Diferença (m) \\
\hline \multirow{4}{*}{1} & Sem parâmetros & 904,451 & 0,087 \\
\cline { 2 - 4 } & NGS relativo & 904,360 & $-0,004$ \\
\cline { 2 - 4 } & NGS absoluto & 904,360 & $-0,004$ \\
\hline \multirow{4}{*}{2} & Sem parâmetros & 903,573 & 0,063 \\
\cline { 2 - 4 } & NGS relativo & 903,510 & 0,000 \\
\cline { 2 - 4 } & NGS absoluto & 903,510 & 0,000 \\
\hline \multirow{3}{*}{3} & Sem parâmetros & 900,396 & 0,057 \\
\cline { 2 - 4 } & NGS relativo & 900,338 & $-0,000$ \\
\cline { 2 - 4 } & NGS absoluto & 900,339 & $-0,000$ \\
\hline
\end{tabular}

Observando a Tabela 8 verifica-se que as diferenças obtidas entre as altitudes obtidas sem parâmetros e com parâmetros da BCAL/UFPR é da ordem de centímetros, diferença já esperada. O maior valor foi alcançado para o ponto 1 e é igual a $8,7 \mathrm{~cm}$. Também se percebe que a maior discrepância entre as altitudes obtidas com os parâmetros da BCAL/UFPR com os do NGS é de $4 \mathrm{~mm}$ para o ponto 1 .

Diante dos resultados da Tabela 8 confirma-se o resultado consagrado a respeito da importância da utilização no processamento dos parâmetros de calibração de antenas, visto que a diferença média obtida foi de $6,9 \mathrm{~cm}$, quando comparadas as coordenadas obtidas com e sem o emprego destes parâmetros de calibração.

A Tabela 9 apresenta os resultados obtidos para as linhas de bases médias (em torno de $45 \mathrm{~km}$ ). São apresentadas as altitudes elipsoidais dos pontos 4, 5 e 6, assim como as diferenças calculadas entre os resultados obtidos, com o emprego das combinações lineares $\mathrm{L}_{1}+\mathrm{L}_{2}$ e $\mathrm{L}_{3}$. 
Tabela 9: Altitudes elipsoidais e as diferenças calculadas para os pontos situados em torno de 45 km de distância com diferentes combinações lineares.

\begin{tabular}{|c|c|c|c|c|}
\hline Ponto & $\begin{array}{c}\text { Combinação } \\
\text { linear }\end{array}$ & Parâmetro & $\begin{array}{c}\text { Altitude } \\
\text { elipsoidal (m) }\end{array}$ & Diferença (m) \\
\hline \multirow{3}{*}{4} & \multirow{9}{*}{$\mathrm{L}_{3}$} & Sem parâmetros & 817,542 & 0,112 \\
\hline & & NGS relativo & 817,462 & 0,032 \\
\hline & & NGS absoluto & 817,461 & 0,031 \\
\hline \multirow{3}{*}{5} & & Sem parâmetros & 914,570 & 0,060 \\
\hline & & NGS relativo & 914,508 & $-0,001$ \\
\hline & & NGS absoluto & 914,508 & $-0,001$ \\
\hline \multirow{3}{*}{6} & & Sem parâmetros & 1097,584 & 0,058 \\
\hline & & NGS relativo & 1097,522 & $-0,004$ \\
\hline & & NGS absoluto & 1097,525 & $-0,002$ \\
\hline \multirow{3}{*}{4} & \multirow{9}{*}{$\mathrm{L}_{1}+\mathrm{L}_{2}$} & Sem parâmetros & 817,528 & 0,071 \\
\hline & & NGS relativo & 817,445 & $-0,012$ \\
\hline & & NGS absoluto & 817,445 & $-0,012$ \\
\hline \multirow{3}{*}{5} & & Sem parâmetros & 914,585 & 0,062 \\
\hline & & NGS relativo & 914,522 & $-0,001$ \\
\hline & & NGS absoluto & 914,523 & $-0,001$ \\
\hline \multirow{3}{*}{6} & & Sem parâmetros & 1097,590 & 0,065 \\
\hline & & NGS relativo & 1097,534 & 0,009 \\
\hline & & NGS absoluto & 1097,534 & 0,009 \\
\hline
\end{tabular}

Quando comparados os resultados entre o emprego das diferentes combinações lineares $\left(\mathrm{L}_{3}\right.$ e $\mathrm{L}_{1}+\mathrm{L}_{2}$ ), verifica-se que houve diferenças para a solução sem parâmetros, que aumentaram (ponto 5 e 6) e que diminuíram (ponto 4) quando comparados os resultados $\mathrm{L}_{3}$ com os $\mathrm{L}_{1}+\mathrm{L}_{2}$. $\mathrm{A}$ diferença, que se destaca das demais é a do ponto 4, sem parâmetros. A diferença, que para a combinação linear $\mathrm{L}_{3}$ era de $11,2 \mathrm{~cm}$, para a outra combinação linear $\left(\mathrm{L}_{1}+\mathrm{L}_{2}\right)$ foi de $7,1 \mathrm{~cm}$. Este ponto alcançou diferenças da ordem de $\mathrm{cm}$ em todos os processamentos, o que pode indicar algum problema no seu processamento. Nos pontos 5 e 6 , onde foram utilizadas as antenas LEIAX 1202 GG e LEIAX 1203+ GNSS, respectivamente, as diferenças com os parâmetros do NGS foram da ordem de mm.

A Tabela 10 apresenta os resultados obtidos para as linhas de bases longas (em torno de $105 \mathrm{~km}$ ). São apresentadas as altitudes elipsoidais dos pontos 7, 8 e 9, assim como as diferenças calculadas entre os resultados obtidos. 
Tabela 10: Altitudes elipsoidais e as diferenças calculadas para os pontos situados em torno de 105 km de distância.

\begin{tabular}{c|c|c|c}
\hline Ponto & Parâmetro & Altitude elipsoidal (m) & Diferença (m) \\
\hline \multirow{4}{*}{7} & Sem parâmetros & 678,605 & 0,058 \\
\cline { 2 - 4 } & NGS relativo & 678,541 & $-0,002$ \\
\cline { 2 - 4 } & NGS absoluto & 678,542 & $-0,001$ \\
\hline \multirow{4}{*}{8} & Sem parâmetros & 807,566 & 0,085 \\
\cline { 2 - 4 } & NGS relativo & 807,480 & $-0,001$ \\
\cline { 2 - 4 } & NGS absoluto & 807,480 & $-0,001$ \\
\hline \multirow{4}{*}{9} & Sem parâmetros & 806,581 & 0,056 \\
\cline { 2 - 4 } & NGS relativo & 806,517 & $-0,009$ \\
\cline { 2 - 4 } & NGS absoluto & 806,519 & $-0,007$ \\
\hline
\end{tabular}

Sem a aplicação de qualquer parâmetro de calibração de antenas GPS, obteve-se uma diferença média de $7,3 \mathrm{~cm}$. As menores diferenças do NGS relativo foram calculadas para o ponto 8, que empregou a antena LEIATX 1230. Este ponto obteve o menor valor de efeito de multicaminho em $\mathrm{MP}_{1}$, com valor igual a $0,245 \mathrm{~m}$. Os pontos 7 e 9, que obtiveram efeito de multicaminho entre $0,266 \mathrm{~m}$ e $0,330 \mathrm{~m}$, alcançaram diferenças superiores a $1 \mathrm{~mm}$.

Visto que para os pontos com linha de base média (em torno $45 \mathrm{~km}$ ) foram realizados processamentos com as combinações lineares $\mathrm{L}_{1}+\mathrm{L}_{2}$ e $\mathrm{L}_{3}$, a fim de selecionar os melhores resultados para cada um destes pontos, foram verificados alguns resíduos remanescentes das Duplas Diferenças de Fase (DDF). Optou-se por analisar as observações GPS do ponto 4 em razão do mesmo ter alcançado as maiores diferenças. As médias das DDF dos satélites GPS, na onda portadora $L_{1}$, para sem parâmetros, no ponto 4 podem ser visualizadas na Figura 2.

Observa-se que as maiores diferenças encontram-se para os PRNs 8 e 29. Verifica-se também que, quando empregada a combinação linear $\mathrm{L}_{1}+\mathrm{L}_{2}$, os resíduos da DDF na portadora $\mathrm{L}_{1}$, advindos das observações dos PRNs 2 e 4, foram maiores que quando utilizada a combinação linear $\mathrm{L}_{3}$. Verifica-se que, quando foi empregada a combinação linear $\mathrm{L}_{1}+\mathrm{L}_{2}$ e a combinação linear $\mathrm{L}_{3}$, a média dos resíduos da DDF do PRN8 foi, respectivamente, de $-3,4 \mathrm{~cm}$ e $-20,4 \mathrm{~cm}$. 


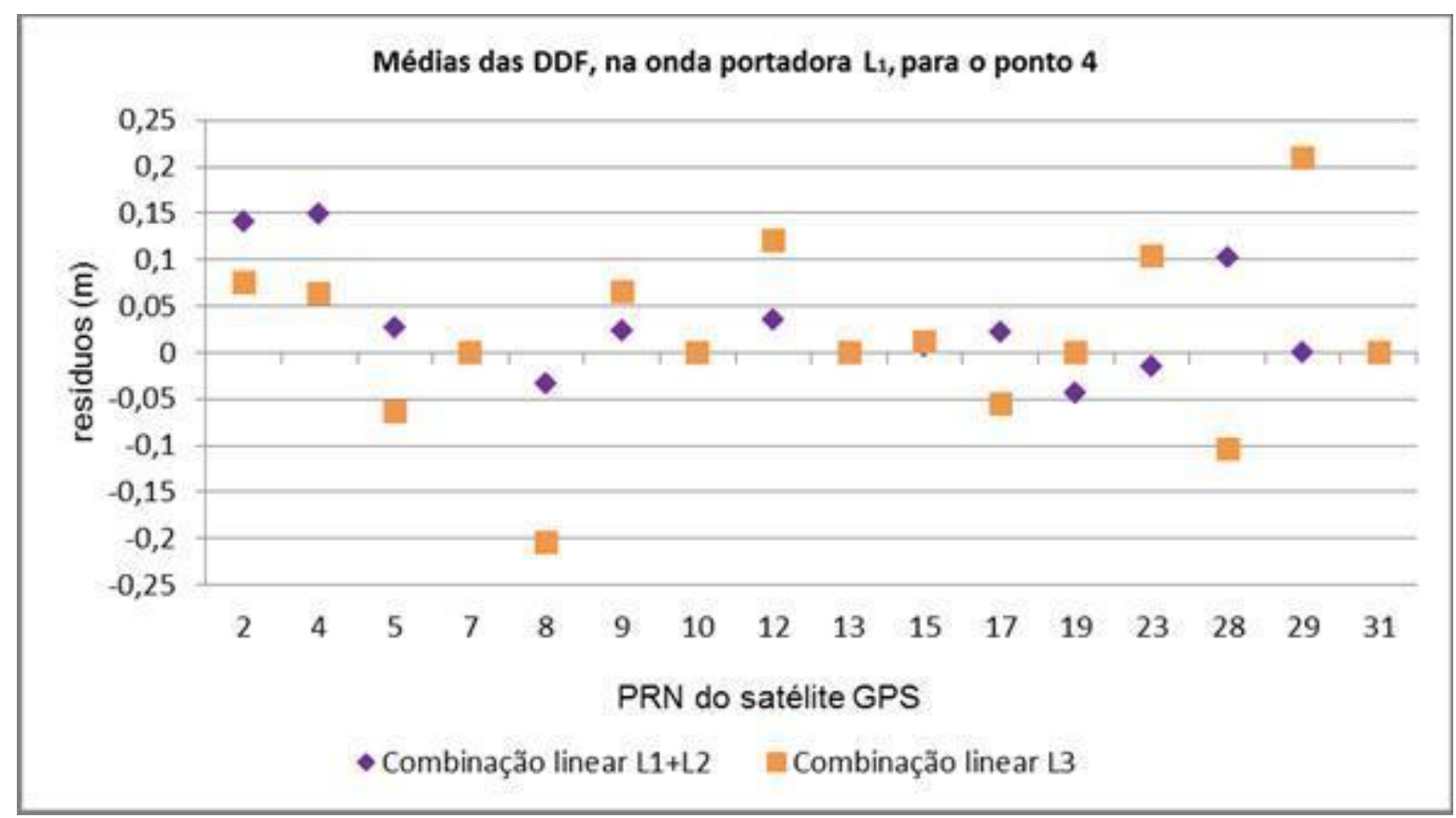

Figura 2: Médias das DDF, na portadora $\mathrm{L}_{1}$, para o ponto 4 (em torno de $45 \mathrm{~km}$ ).

Nas Figuras 3 e 4 visualizam-se os resíduos das DDF, na onda portadora $\mathrm{L}_{1}$, do PRN8 citado anteriormente. Nota-se que os resíduos foram calculados entre o satélite de referência (PRN27) e o PRN8 observado durante o período de levantamento no ponto 4. Observa-se que, quando da aplicação da combinação linear $\mathrm{L}_{1}+\mathrm{L}_{2}$ os resíduos variam de $-6 \mathrm{~cm}$ à $-14 \mathrm{~cm}$, enquanto que com a outra combinação linear $\left(\mathrm{L}_{3}\right)$, a variação é de $-11 \mathrm{~cm}$ à $-27 \mathrm{~cm}$. Portanto, para este ponto, a melhor solução resultou do emprego da combinação linear $\mathrm{L}_{1}+\mathrm{L}_{2}$.

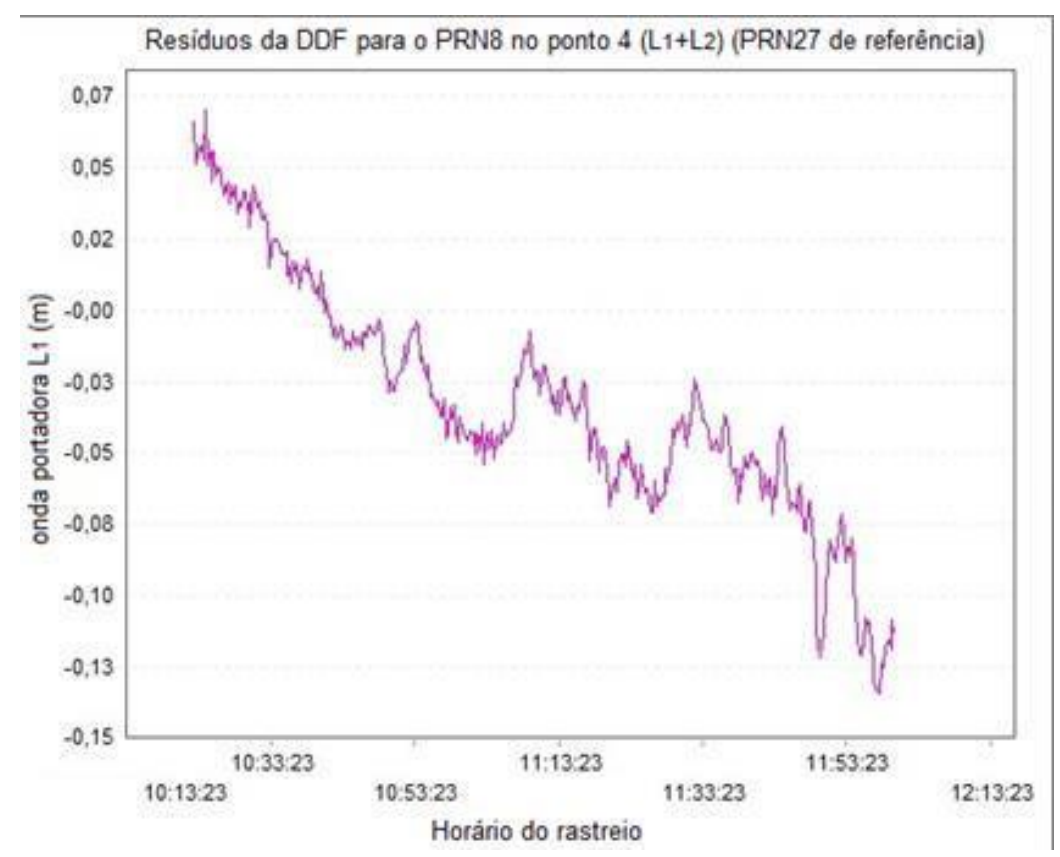

Figura 3: Resíduos das DDF (PRN8), na onda portadora $\mathrm{L}_{1}$, com combinação linear $\mathrm{L}_{1}+\mathrm{L}_{2}$, para o ponto 4 (em torno de $45 \mathrm{~km})$. 


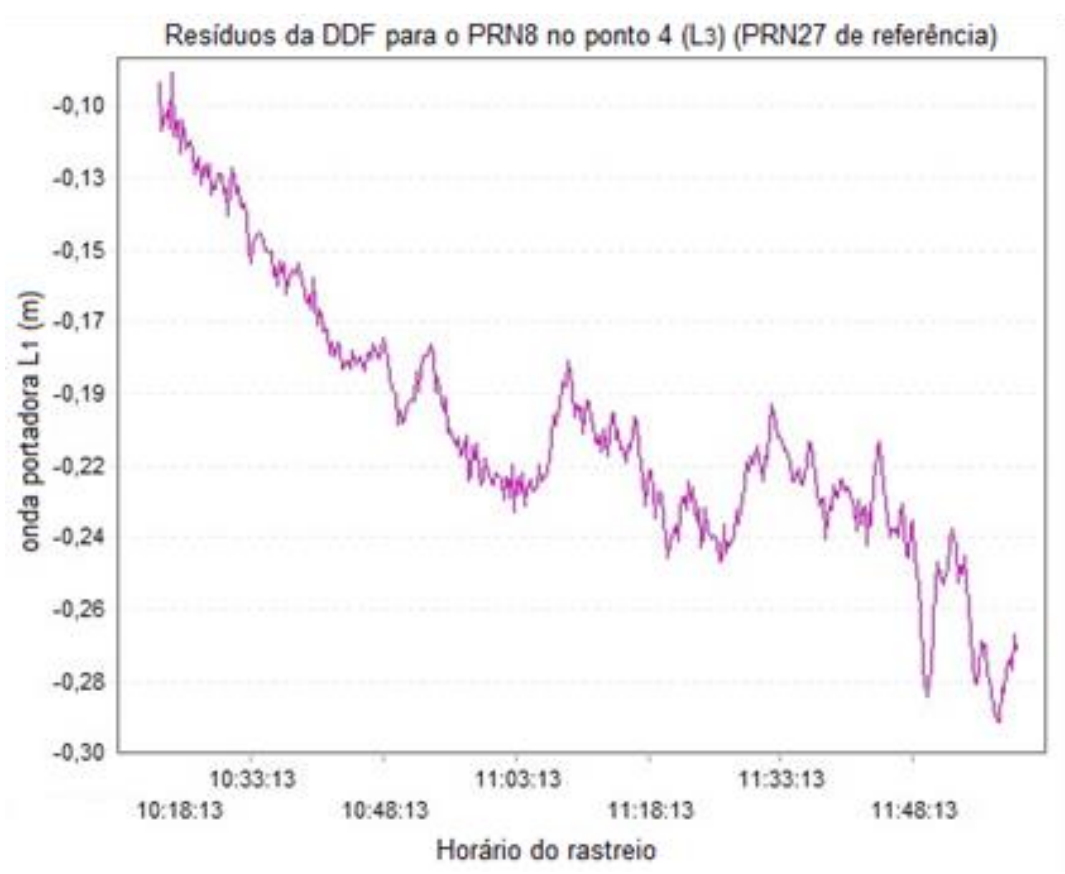

Figura 4: Resíduos das DDF (PRN8), na onda portadora $\mathrm{L}_{1}$, com combinação linear $\mathrm{L}_{3}$, para o ponto 4 (em torno de $45 \mathrm{~km}$ ).

Para melhor comparar as diferenças das altitudes elipsoidais, a Tabela 11 apresenta as diferenças para todos os resultados selecionados, assim como a combinação linear empregada e a solução das ambiguidades obtida.

Tabela 11: Diferenças das altitudes elipsoidais dos pontos 1 a 9.

\begin{tabular}{|c|c|c|c|c|c|c|}
\hline \multirow[b]{2}{*}{ Ponto } & \multirow{2}{*}{$\begin{array}{l}\text { Número de } \\
\text { satélites GPS }\end{array}$} & \multirow{2}{*}{$\begin{array}{c}\text { Solução das } \\
\text { ambiguidades }\end{array}$} & \multirow{2}{*}{$\begin{array}{c}\text { Combinação } \\
\text { linear }\end{array}$} & \multicolumn{3}{|c|}{ Diferença da altitude (m) } \\
\hline & & & & $\begin{array}{c}\text { Sem } \\
\text { parâmetros }\end{array}$ & \begin{tabular}{|c|} 
NGS \\
relativo
\end{tabular} & $\begin{array}{c}\text { NGS } \\
\text { absoluto }\end{array}$ \\
\hline 1 & 15 & Fixa & $\mathrm{L}_{1}+\mathrm{L}_{2}$ & 0,087 & $-0,004$ & $-0,004$ \\
\hline 2 & 15 & Fixa & $\mathrm{L}_{1}+\mathrm{L}_{2}$ & 0,063 & 0,000 & 0,000 \\
\hline 3 & 14 & Fixa & $\mathrm{L}_{1}+\mathrm{L}_{2}$ & 0,057 & $-0,000$ & $-0,000$ \\
\hline 4 & 15 & Fixa & $\mathrm{L}_{1}+\mathrm{L}_{2}$ & 0,071 & $-0,012$ & $-0,012$ \\
\hline 5 & 15 & Flutuante & $\mathrm{L}_{3}$ & 0,060 & $-0,001$ & $-0,001$ \\
\hline 6 & 15 & Flutuante & $\mathrm{L}_{3}$ & 0,058 & $-0,004$ & $-0,002$ \\
\hline 7 & 16 & Flutuante & $\mathrm{L}_{3}$ & 0,058 & $-0,002$ & $-0,001$ \\
\hline 8 & 11 & Flutuante & $\mathrm{L}_{3}$ & 0,093 & 0,012 & 0,012 \\
\hline 9 & 16 & Flutuante & $\mathrm{L}_{3}$ & 0,056 & $-0,009$ & $-0,007$ \\
\hline
\end{tabular}

As diferenças do NGS relativo e absoluto são semelhantes em todos os pontos com linhas de base em torno de $9 \mathrm{~km}$. Os pontos 2 e 3 não obtiveram diferenças. Os pontos 4 e 8 , com linhas de base em torno de $45 \mathrm{~km}$ e $105 \mathrm{~km}$, respectivamente, se destacam por apresentarem diferenças do NGS relativo e absoluto maiores que $1 \mathrm{~cm}$. Nestes pontos a antena empregada foi a mesma LEIATX 1230 (Tabela 1).

Observa-se que, sem a utilização dos parâmetros, as maiores diferenças ocorrem nos pontos 1, 4 e 8. É possível, através da Figura 5 visualizar graficamente os valores das diferenças apresentadas na Tabela 11 . 


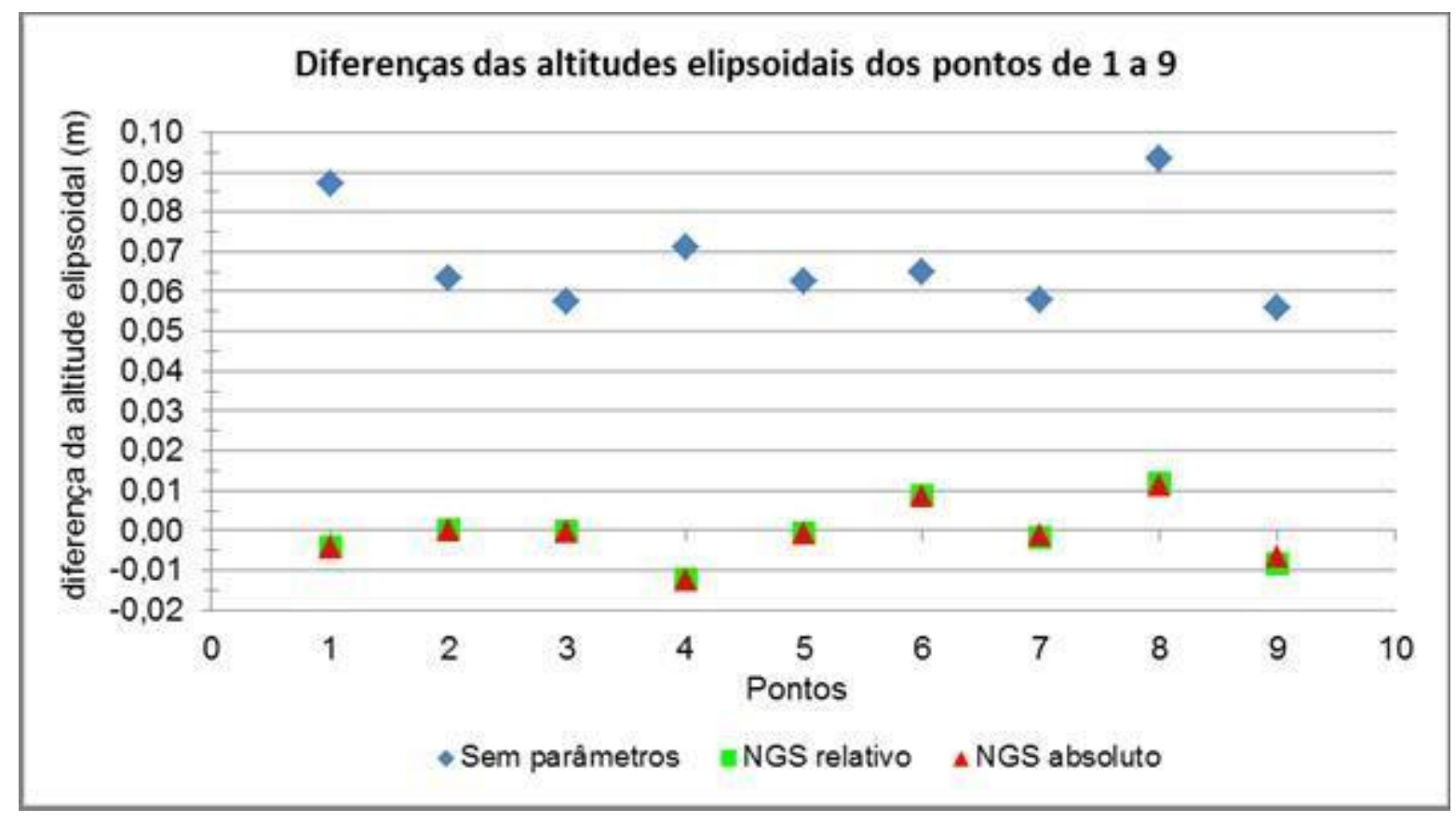

Figura 5: Diferenças das altitudes elipsoidais dos pontos 1 a 9.

Observou-se que, quando não foram empregados os PCO-PCV, obteve-se uma diferença média, na altitude elipsoidal, para todos os pontos, de $6,6 \mathrm{~cm}$ em relação à aplicação dos parâmetros da BCAL/UFPR. Após análise dos valores das altitudes elipsoidais e das diferenças encontradas ressalta-se a importância da aplicação dos parâmetros de calibração de antenas GPS. Verificouse que, com a inserção dos parâmetros médios do NGS (relativo e absoluto) as altitudes elipsoidais foram diferentes das que utilizaram os parâmetros individuais da BCAL/UFPR. Isto comprova também que há consistência entre os parâmetros do NGS. Em cinco pontos as diferenças do NGS relativo e absoluto foram maiores que $2 \mathrm{~mm}$. Isto indica que quando se almeja acurácia milimétrica pode ser necessário o uso dos parâmetros próprios das antenas utilizadas.

\section{CONCLUSÕES}

Destaca-se que os resultados da BCAL/UFPR advêm da utilização de parâmetros individuais, portanto, fiéis às antenas utilizadas. Os valores PCO-PCV, oriundos do NGS, são parâmetros médios fornecidos para cada modelo de antena.

Mediante os resultados alcançados para as altitudes elipsoidais, pode-se afirmar que os parâmetros de calibração de antenas GPS são de suma importância na obtenção da alta acurácia no posicionamento relativo GPS. Foram encontradas diferenças na ordem de centímetros quando não se utilizou parâmetros, o que contribui para a degradação da acurácia do posicionamento realizado.

Dos pontos de linhas de base curta (em torno de $9 \mathrm{~km}$ ), somente o ponto 1 obteve diferença, da ordem de mm, quando comparadas as altitudes elipsoidais com os parâmetros da BCAL/UFPR e 
os médios do NGS (relativo e absoluto). Nas linhas de bases médias e longas, verificaram-se diferenças de $3 \mathrm{~mm}$ a 1,2 $\mathrm{cm}$ em alguns pontos (4, 6, 8 e 9) quando da comparação das altitudes elipsoidais com o emprego dos parâmetros próprios da BCAL/UFPR e com os parâmetros médios NGS absoluto e relativo. Tal fato indica que para $67 \%$ dos pontos rastreados, com linhas de bases médias e longas, o emprego dos parâmetros individuais das antenas GPS foi necessário para o alcance de alta acurácia no posicionamento relativo.

Os resultados também indicam que através do emprego de um programa comercial de processamento de dados GPS, um profissional que dispõe somente desse ambiente computacional e que, mesmo assim almeja alta acurácia em seu projeto, se beneficiaria do uso dos parâmetros próprios de calibração de antenas.

\section{AGRADECIMENTOS}

Ao Conselho Nacional de Desenvolvimento Científico e Tecnológico (CNPq), ao Programa de Pós-Graduação em Ciências Geodésicas (PPGCG), ao Laboratório de Geodésia Espacial e Hidrografia (LAGEH) e à Universidade Federal do Paraná (UFPR).

Este trabalho tem financiamento da Coordenação de Aperfeiçoamento de Pessoal de Nível Superior (Capes) através do projeto número 103/2012, intitulado Otimização de Redes Geodésicas Empregando Estações GPS com Antenas com Parâmetros Próprios Conhecidos.

\section{REFERÊNCIAS BIBLIOGRÁFICAS}

Pinto, E. B.; Chuerubim, M. L. Discrepâncias Existentes Entre as Coordenadas Estimadas pelos Métodos de Posicionamento Relativo e Absoluto Utilizando Receptores de Simples Frequência: Estudo de Caso Rede GNSS Local da Universidade Federal de Uberlândia (UFU). In: Anais XVII Simpósio Brasileiro de Sensoriamento Remoto, João Pessoa, Paraíba, 25-29 de abril, 2015.

Huinca, S. C. M. Calibração Relativa de Antenas GNSS na BCAL/UFPR. Dissertação (Mestrado em Ciências Geodésicas). Curso de Pós-Graduação em Ciências Geodésicas, Setor de Ciências da Terra, Departamento de Geomática, Universidade Federal do Paraná, 2009.

Huinca, S. C. M.; Krueger, C. P. Determinação de Parâmetros de Calibração Relativos em Nível Absoluto de Antenas de Receptores GPS. Revista Brasileira de Cartografia, $n^{\circ} 63$ Ed. Especial Geodésia e Topografia. 2011.

Huinca, S. C. M.; Krueger, C. P.; Mayer, M.; Knöpfler A.; Heck, B. First Results of Relative Field Calibration of a GPS Antenna at BCAL/UFPR (Baseline Calibration Station for GNSS Antennas at UFPR/Brazil). In: Geodesy for Planet Earth - 2009 International Association of Geodesy Symposium. New York: Sringer Heidelberg Dordrecht, 2012, v. 136, p. 739-744.

Kersten, T.; Schön, S. Towards Modelling Phase Center Variations for Multi-Frequency and Multi-GNSS. In: $5^{\text {th }}$ ESA Workshop on Satellite Navigation Tecnologies and European Workshop on GNSS Signals and Signal Processing, IEEE Xplore, 8p., ESA/ESTEC, Noordwijk, Netherland, December 08-10, 2010.

Kouba, J. A Guide to Using International GNSS Service (IGS) Products (2009). Acesso em: 22 de Abril de 2015. http://igscb.jpl. nasa.gov/components/usage.html. 
Mader, G. GPS Antenna Calibration at the National Geodetic Survey. GPS Solutions, Vol. 3, $\mathrm{N}^{\circ} 1$, p.50-58, 1999.

Menge, F.; Seeber, G.; Völksen, C. Wübbena, G.; Schmitz, M. Results of absolute field calibration of GPS antenna PCV. In: Proceedings of the $11^{\text {th }}$ International Technical Meeting of the Satellite Division of the Institute of Navigation ION GPS-98, Nashville, Tennessee, September 15-18, 1998.

Santos, D. P.; Faggion, P. L.; Graça, N. L. S. S.; Kanke, R.; Quirino, R. A.; Holler, W. A. Determinação da Altitude do Pico do Tucum na Serra do Mar Paranaense Utilizando GPS. In: III Simpósio Brasileiro de Ciências Geodésicas e Tecnologias da Geoinformação, Recife, Pernambuco, 27-30 de Julho, 2010.

Seeber, G. Real-Time Satellite Positioning on the Centimeter Level in the 21st Century using Permanent Reference Stations. Institut für Erdmessung, University of Hannover, 2000.

Seeber, G. Satellite Geodesy: Foundations, Methods, and Applications. Berlin: Walter de Gruyter, 589 p., 2003.

Silva, L. A.; Chuerubim, M. L.; Pires, A. C. G.; Gomes, P. G. Análise da Qualidade Geométrica da Rede GNSS Local da UFU Campus Santa Mônica. Revista Eletrônica: Tempo - Técnica Território, v. 4, p. 29-46, 2013.

UNAVCO (University NAVSTAR Consortium). TEQC - Tutorial. Acesso em: 03 de Setembro de 2013. http://facility.unavco.org/software/teqc/tutorial.html.

Wübbena, G.; Menge, F.; Schmitz, M.; Seeber, G.; Völksen, C. A. New Approach for Field Calibration of Absolute Antenna Phase Center Variations. Presented at ION GPS-96, Kansas City, Missouri, USA, 1205-1214, 1996.

Recebido em janeiro de 2015. Aceito em abril de 2015. 